Journal of Engineering and Applied Sciences 14 (4): 1274-1278, 2019

ISSN: 1816-949X

(C) Medwell Journals, 2019

\title{
Internet of Things Utilization for eHealthcare Monitoring
}

\author{
Shokhan M. Al-Barzinji and Thaaer Kh. Asman \\ College of Computer Science and Information Technology, University of Anbar, Anbar, Iraq \\ College of Art, University of Anbar, Anbar, Iraq
}

\begin{abstract}
Wireless sensor networks play an important role in providing eHealthcare (eHc), data storage processing and sensing devices. The growing technologies in various fields such as WSNs, dedicated computer system (Embedded systems), Nanotechnologies and sensing technology, led to developing intelligent systems for patients telemonitoring to control different medical cases easily. Internet of Thing (IoT) can be considered as a modern tool that recently emerged in health communication systems and it's an important way for modern healthcare in various health applications. The wearable sensor devices can recognize abnormal and unexpected changes in physiological parameters for elderly in order to evaluate the vital signs for medical diagnoses, hence, giving a necessary medication to the patient in a timely manner to avoid critical cases. This study presents a communication gateway (System-On-Chip (SoC)) which controls the sensors based on patient's parameters such as pulse rate sensor, temperature sensor and brain signals coming from the brain. The SoC gained data from outside, process these data, then transmit it over Wireless Networks (WSNs) through a proper protocol in order to provide a suitable telemonitoring healthcare elderly by specialists.
\end{abstract}

Key words: System-On-Chip (SoC), Internet of Thing (IoT), Wireless Sensor Networks (WSNs), eHealthcare (eHc), pplications, sensing devices

\section{INTRODUCTION}

Internet plays an important role in the development of a key footprint in the lives of individuals and peoples such as education, various commercial transactions, entertainment, social networks, shopping and others. Then, IoT is the modern trend of this era (Gubbi et al., 2013). The conceptual point of view, imagine the world a where many objects can communicate, analyze, sense and share private IP or public networks. These objects obtain information or data at particular intervals, explain and necessary response and supply a smart grid for analysis, design, making the decision and answering. This is briefly the idea of IoT based on the gateway, various sensors, and WSN, that allow users to access information and different app. This, in turn, gives more explicit access to EHC. Most of the research developed has focused on improving the healthcare quality provided to patients and the elderly in particular, through the development of communication devices and sensors (David, 2013). Some suggest that the reason for the recent development of these methods is elderly. According to WHO (World
Health Organization) the demands for medical care instruments will be increasing because of increasing elderly population.

As a result of long-term healthcare, especially, consultation with specialists, these medical care devices are expensive and the cost of hospital therapy is high and increases in cases of recovery or surgery. In this time, most of the medical centers or hospitals are seeking to move patients to their homes and provide necessary treatment through telemedicine. Then, the telemedicine was becoming importance and requirement (Vermesan et al., 2013).

Problem statement (aim of study): Through treatments duration, the pathological cases monitor locally are periodical (inside the old house or patient's house) by the specialists as well as giving continuing instructions for patients or necessary treatments. Therefore, increase health awareness for people via. telemedicine gives a great opportunity to monitor the therapeutic progress of patients or older people and to provide them with the best treatment and close surveillance.

Corresponding Author: Shokhan M. Al-Barzinji, College of Computer Science and Information Technology, University of Anbar, Anbar, Iraq 
Table 1: The discriptin of various IEEE protcols

\begin{tabular}{lllll}
\hline Market & ZigBee TM & GSM/GPRS & WI-FI & Bluetooth \\
\hline Name standard & 802.15 .4 & CDMA $\times$ RIT & $802.11 \mathrm{~b}$ & M 802.15.1 \\
App focus & Monitoring & Wide area & Web, Email & Cable replacement \\
& and control & Voice and data & Video & $250 \mathrm{kB}+$ \\
System resources & $4-32 \mathrm{kB}$ & $16 \mathrm{MB}+$ & $1 \mathrm{MB}+$ & 1.7 \\
Battery life (days) & $100-1.000+$ & 1.7 & 3.5 & 7 \\
Network size & Unlimited $\left(2^{6+}\right)$ & 1 & $11.000+$ & 720 \\
Bandwidth $(\mathrm{kB} / \mathrm{sec})$ & $20-250$ & $64-128+$ & 1.100 & $1-10+$ \\
Transmission & $1-100+$ & $1.000+$ & & \\
Range(m) & & & Speed, & Cost \\
Success metrics & Reliability, & Reach, & Flexibilty & Convenience \\
\hline
\end{tabular}

\section{Literature review}

Healthcare surveillance system: In this study, in order to patient's monitoring closely, the standard of ZigBee that operates on the IEEE 802.15.4 protocol physical radio specification for wireless patient's surveillance was used. ZigBee module used to monitor patients closely in both indoor/outdoor locations to transmit ECG, EEG signals real time to nearest emergency center for prompt treatment. In all healthcare surveillance systems, there is an alarming circuit (SoC-based) used to notify the specialist or doctor through sensor network if the reading of blood pressure, temperature, abnormal EEG, abnormal ECG goes out of the standard limit for a patient or elderly, for example, in blood pressure monitoring systems, the blood pressure can be computed by using a technique of PPG (photoplethysmography) and volume oscillometric continuously (Ram, 2013).

Wireless Sensor Networks (WSNs): WSNs is a set of sensors that are utilized to tracking or transferring a specific physical or chemical phenomenon (such as heat, humidity, vibration, light, etc.) and then transmit information about this phenomenon wirelessly to a server center data to take advantage without a need for the patient presence locally. Sensor networks can extremely benefit from web services. These services allow allows the utilize of sensor networks in all systems that are an integral part of IT components such as healthcare monitoring systems, home automation etc. (Ahmed et al., 2015). In the IoT-based healthcare surveillance systems all data are gathering from various sensors anytime and anywhere, these sensors are directly connected to the network. The collected data in the main station have become a huge data because of continuous storage, then these data need compression the space to store more data. In case of data transmission through WSNs for remote healthcare surveillance app, the total bandwidth not used, effectively. This is lead to lost most data transferred because of delay, this status is unacceptable in healthcare systems (Pande and Padwalkar, 2014). The sensor networks architecture play an important role in healthcare systems in the handover of continuous symptoms of chronic patients to the specialists. The sensors operation method depends entirely on the method of selecting the network for these sensors. The suitable network has selected depend on the energy consumption, accessibility, cost, easy to the configuration, network performance and bandwidth. A Table 1 show all IEEE protocols available comparison (Jara et al., 2013).

There is a kind of computer network called PAN (Personal Area Networks) follows IEEE 802.15 standard, created by ZigBee from tiny, LPDR (Lower-Power Digital Radios). These networks used to communicate between computers close to the patient or the elderly (such as mobile devices, smartphone and PDAs). PANs used for short-range wireless data transfer at relatively low costs and permits short-range data to reach via. a longer distance by crossing it via. sporadic devices or nodes to leave more distant. This is led to low power consumption because of the lower data rate. In Table 1 description of IEEE standard comparison (Santos et al., 2014).

The technique that is similar to technique ZigBee in its function is Bluetooth technology because have a protocol based on master-slave structure. The Bluetooth devices are connected to each other by using unlicensed short-scale radio frequency. The standard of Wi-Fi that is operates on the IEEE protocol that is used to transfer all data wirelessly used to raising the communication range up to $5 \mathrm{~km}$ distance. WiMAX is a wireless MAN technology that allows for broadband communication up to $15 \mathrm{~km}$ (Collier, 2015).

\section{MATERIALS AND METHODS}

Networks limitations: The major limitations of ZigBee include: May not have the ability to transmit vital signals to patients or elderly through emergency messages. 
These vital signs are very important because they convey the patient's health status to the doctor in order to direct the appropriate therapy (Abdulbaqi et al., 2017) .

- Short range

- Low complexity

- Low data speed

Due to their high cost, GSM and GPRS are normally used in concentrators to transmit data to the main station or in high-end multi-function meters (Clifton et al., 2014).

\section{RESULTS AND DISCUSSION}

Healthcare networks-based IoT: IoT is one of the essential vital components and the backbone of the Internet, through which the necessary medical information is transferred to patients or the elderly in order to enable the necessary healthcare for them (Kiran et al., 2014).

The concept of the internet of things is collect available technologies, connecting humans via. World Wide Web and take this connection in order to facilitate the connection of devices to the Internet as well as connecting devices to human and connect the machine to machine easily. In order to understand more about the IoT, identify the basic building blocks of IoT (Mukhopadhyay, 2015):

Sensor technology: Used to sensing the different information from position weather, network parameters, and health data for patients.

The Gateway is a gate to build a bridge between the external internet and internal network or World Wide Web by aggregating various data from various sensor nodes and transmit these data to the infrastructure of the internet (Kavitha and Perumalraja, 2014).

Big data and infrastructure of cloud/server: The various data can be transmitted through the gateway, stored and processed within the infrastructure of the cloud by using the engine of big data analysis and then use this data to implement smart actions to make these devices intelligent devices (Malhi et al., 2012).

Mobile Apps (End user): The mobile apps used to help patients or elderly to monitor and control him vital parameters and control their room's thermostat in order to send actual reports to smart devices.

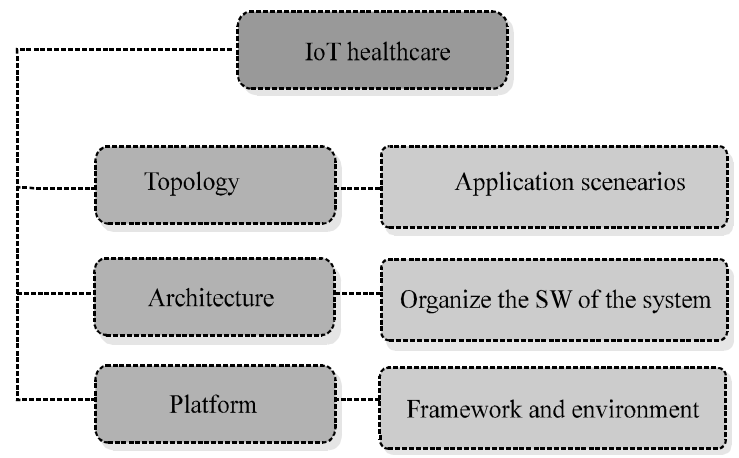

Fig. 1: IoT Healthcare issues network

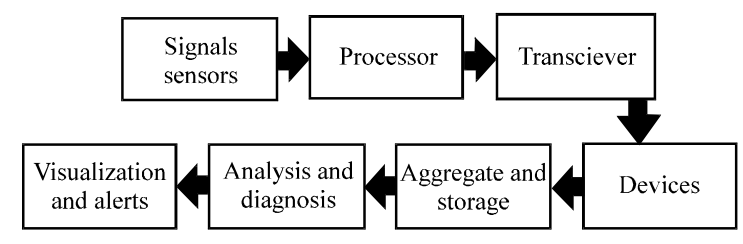

Fig. 2: General architecture of human healthcare monitoring system

Ipv6: it's a backbone of the IoT. The IP addresses are related to the only internet if you are the user. With IPv6, having $3.4^{*} 10^{38} \mathrm{IP}$ addresses. Figure 1 is the figure of the issues of IoT healthcare network.

System description: The system of human healthcare monitoring or can be described by the following general architecture (Fig. 2) each parameter has a sensor to be monitored. Body temperature, ECG signals, EEG signals, etc. can be measured by various sensors. The raw data collected from the various sensors are processed and displayed on the screen by the processor.

If the feature of data transmitted wirelessly is on a trans-receiver can be used to send/receive data to/from the central station. At the sensing phase, the raw data are completely processed, stored in the device memory and then display directly in graphical or numerical format. In this study, the proposed system architecture can be shown as the Fig. 3.

The system consists of multi-sensors (ECG sensors, EEG sensors, body temperature sensors, blood sugar level, etc). The SoC (microcontroller) will collect all various data from multi-sensors to analyze these data and process. After that, all processed data are transmitted through Wi-Fi protocol to the internet. The traveled data are stored on the main servers. The processed data are showing on a website. The role of specialists or doctors are accessing the website through unique IP address in the internet browser anywhere anytime and read the patients reports real time. 


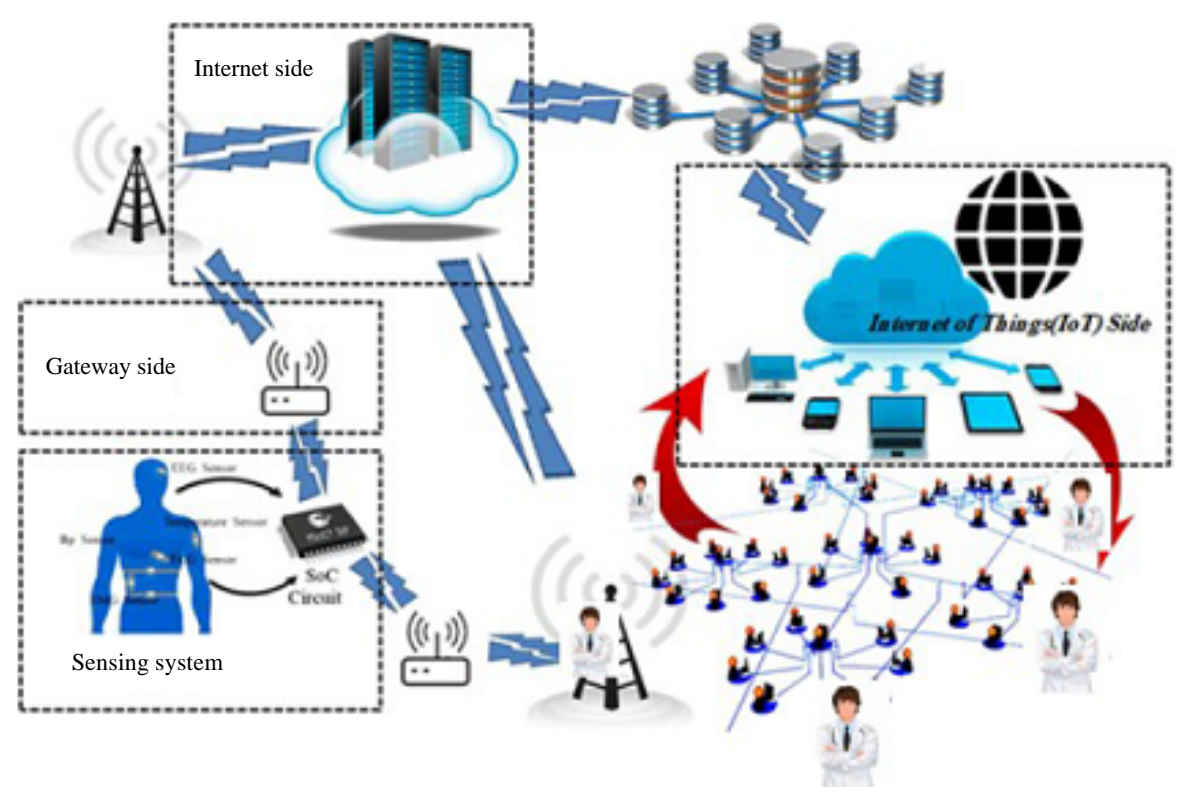

Fig. 3: Infrastructure of $(\mathrm{eHc})$ surveillance system

\section{CONCLUSION}

In this study, a Human (eHc) monitoring system using the Internet of Things (IoT) is presented. The IoT is essential in healthcare due to: Develop the horizon all types of sensor technology (fixed and mobile devices), and provide a necessary healthcare for patients and elderly real time by collecting a necessary data for patients or elderly periodically $24 / 7 \mathrm{~h} /$ day for a diagnosis and therapy rapidly. Different data collection automatically continuously reduces the chance of manual intervention of humans in the collection of these data and thus reduce the probability of error and reduce costs and increase efficiency.

\section{REFERENCES}

Abdulbaqi, A.S., 2017. Smartphones Impact in medical marketing and $m$-healthcare for developing countries. J. Eng. Appl. Sci., 12: 6057-6061.

Ahmed, M.U., H. Banaee, X. Rafael-Palou and A. Loutfi, 2015. Intelligent Healthcare Services to Support Health Monitoring of Elderly. In: Internet of Things, Giaffreda, R. (Ed.). Springer, Berlin, Germany, ISBN:978-3-319-19655-8, pp: 178-186.

Clifton, L., D.A. Clifton, M.A. Pimentel, P.J. Watkinson and L. Tarassenko, 2014. Predictive monitoring of mobile patients by combining clinical observations with data from wearable sensors. IEEE. J. Biomed. Health Inf., 18: 722-730.
Collier, S.E., 2015. The emerging enernet: Convergence of the smart grid with the internet of things. Proceedings of the 2015 IEEE International Conference on Rural Electric Power (REPC), April 19-21, 2015, IEEE, Asheville, North Carolina, ISBN:978-1-4799-7555-6, pp: 65-68.

David, N., 2013. How the Internet of Things is Revolutionizing Healthcare. Freescale Semi conductor, Austin, Texas, USA.,.

Gubbi, J., R. Buyya, S. Marusic and M. Palaniswami, 2013. Internet of Things (IoT): A vision, architectural elements and future directions. Future Generation Comput. Syst., 29: 1645-1660.

Jara, A.J., M.A. Zamora-Izquierdo and A.F. Skarmeta, 2013. Interconnection framework for mHealth and remote monitoring based on the internet of things. IEEE. J. Sel. Areas Commun., 31: 47-65.

Kavitha, K.C. and R. Perumalraja, 2014. Smart wireless healthcare monitoring for drivers community. Proceedings of the 2014 International Conference on Communications and Signal Processing (ICCSP), April 3-5, 2014, IEEE, Melmaruvathur, India, ISBN:978-1-4799-3357-0, pp: 1105-1108.

Kiran, M.S., P. Rajalakshmi, K. Bharadwaj and A. Acharyya, 2014. Adaptive rule engine based IoT enabled remote health care data acquisition and smart transmission system. Proceedings of the 2014 IEEE International Conference on World Forum Internet of Things (WF-IoT), March 6-8, 2014, IEEE, Seoul, South Korea, ISBN:978-1-4799-3459-1, pp: 253-258. 
Malhi, K., S.C. Mukhopadhyay, J. Schnepper, M. Haefke and H. Ewald, 2012. A Zigbee-based wearable physiological parameters monitoring system. IEEE Sens. J., 12: 423-430.

Mukhopadhyay, S.C., 2015. Wearable sensors for human activity monitoring: A review. IEEE. Sens. J., 15: 1321-1330.

Pande, P. and A.R. Padwalkar, 2014. Internet of things a future of internet: A survey. Intl. J., 2: 354-361.

Ram, S., 2013. Internet-of-Things (IoT) advances home healthcare for seniors. ADLINK, New Taipei, Taiwan. http://www.embeddedintel.com/special_features.ph p?article $=2721$
Santos, A., J. Macedo, A. Costa and M.J. Nicolau, 2014. Internet of things and smart objects for m-health monitoring and control. Procedia Technol., 16: 1351-1360.

Vermesan, O., P. Friess, P. Guillemin, H. Sundmaeker and M. Eisenhauer et al., 2013. Internet of Things Strategic Research and Innovation Agenda. In: Internet of Things (IoT) Converging Technologies for Smart Environment and Integrated Ecosystems, Vermesan, O.P.F. (Ed.). River Publishers, Aalborg, Denmark, ISBN:978-87-9298273-5, pp: 153-343. 